\title{
Percepciones en la vida diaria del paciente con poliquistosis renal autosómica dominante
}

\author{
Marta Beatriz Luque Llaosa, Amaranta Fuenmayor Díaz, Anna Matamala Gastón, Esther Franquet Barnils \\ Fundación Puigvert. Barcelona. España
}

\section{Resumen}

Introducción: La Poliquistosis Renal Autosómica Dominante (PQRAD) es la enfermedad renal hereditaria más frecuente, constituye la tercera causa de enfermedad renal crónica terminal (ERCT) en edad adulta.

Objetivo: Conocer y comprender la experiencia vital de las personas con PQRAD y las repercusiones sentidas en todos los ámbitos de su vida cotidiana, desde una perspectiva bio-psico-social y espiritual, para ofrecer unos cuidados enfermeros de calidad.

Material y Método: Estudio cualitativo basado en el paradigma metodológico de la Teoría Fundamentada de Glaser y Strauss y en el paradigma constructivista.

Los participantes fueron personas diagnosticadas de $P Q R A D$, que se encontraban en fases previas al inicio de terapia renal sustitutiva. El tamaño de la muestra vino dado por la saturación de los datos y fue de 12 participantes que formaron parte de dos grupos focales. Las conversaciones fueron grabadas en audio y trascritas para el análisis posterior de su contenido.

Técnica: Grupo Focal. Se realizaron dos grupos de una media de dos horas de duración, con la presencia de un moderador y dos observadores.

Resultados: Emergieron cinco grandes categorías conceptuales: vivencia/mecanismos de afrontamiento, repercusiones del factor hereditario, síntomas físicos y calidad de vida, perspectivas de futuro y expectativas del entorno sanitario.

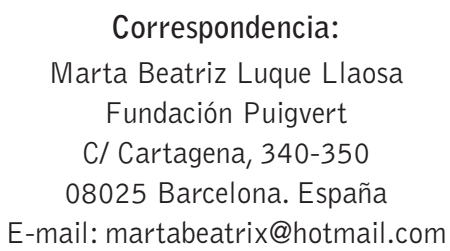

Correspondencia:

Marta Beatriz Luque Llaosa

Fundación Puigvert

C/ Cartagena, 340-350

08025 Barcelona. España

E-mail: martabeatrix@hotmail.com

Conclusiones: La experiencia vital de las personas con PQRAD no presenta un pensamiento único, sino que aparecen puntos de disenso en diversos aspectos que hacen cada experiencia propia e individual. Resulta crucial una atención multidisciplinar desde el momento del diagnóstico, en la que Enfermería apunta como uno de los pilares fundamentales en el acompañamiento, escucha y orientación de las personas con PQRAD y sus familias, desde su rol autónomo y colaborativo.

PALABRAS CLAVE: poliquistosis renal; enfermedad hereditaria; investigación cualitativa; teoría fundamentada; calidad de vida; afrontamiento.

Perceptions in the daily life of the patient with autosomal dominant polycystic kidney disease

\section{Abstract}

Introduction: Autosomal Dominant Polycystic Kidney Disease (ADPKD) is the most common hereditary kidney disease. It is the third cause of end-stage chronic kidney disease (ESRD) in adulthood.

Objective: To know and understand the life experience of people with ADPKD and the repercussions felt in all areas of their daily life, from a bio-psycho-social and spiritual perspective, to offer quality nursing care.

Material and Method: Qualitative study based on the methodological paradigm of the Grounded Theory of Glaser and Strauss and on the constructivist paradigm.

The participants were people diagnosed with ADPKD, who were in phases prior to the initiation of renal replacement therapy. The size of the sample was given by 
the saturation of the data and it was of 12 participants who were part of two focus groups. The conversations were recorded in audio and transcribed for the later analysis of their content.

Technique: Focus Group. Two groups were carried out, of an average of two hours, with the presence of a moderator and two observers.

Results: Five major conceptual categories emerged: experience / coping mechanisms, repercussions of the hereditary factor, physical symptoms and quality of life, future prospects and expectations of the healthcare environment.

Conclusions: The life experience of people with ADPKD does not present a single thought, but there are points of dissent in various aspects that make each experience unique and individual. A multidisciplinary care is crucial from the moment of the diagnosis, in which nursing is one of the fundamental pillars in the accompaniment, listening and orientation of people with ADPKD and their families, from their autonomous and collaborative role.

KEYWORDS: polycystic kidney disease; hereditary disease; qualitative research; grounded theory; quality of life; coping.

\section{Introducción}

La poliquistosis renal autosómica dominante (PQRAD) es la enfermedad renal hereditaria más frecuente. Su prevalencia estimada está muy discutida y oscila entre 1 cada 1000 personas y una de cada 2000. Los pacientes con PQRAD constituyen un 6-10\% de la población en tratamiento renal sustitutivo, siendo por lo tanto, una enfermedad de gran impacto social ${ }^{1}$.

La PQRAD está causada por la mutación de dos genes distintos (PKD1 y PKD2), y representa el 90\% de todos los casos de poliquistosis renal ${ }^{2}$. Se caracteriza por el desarrollo progresivo de quistes renales bilaterales llenos de líquido que, con el tiempo, suelen conducir a la enfermedad renal crónica terminal (ERCT) en la edad adulta, siendo la tercera causa de $\mathrm{ERCT}^{3}$. También se asocia a diversas manifestaciones extrarrenales como poliquistosis hepática, hipertensión arterial, aneurismas intracraneales y anomalías valvulares.
Uno de los principales síntomas es el dolor. El dolor agudo está causado por pielonefritis, infección quística, hemorragia quística y urolitiasis ${ }^{1}$, mientras que la principal causa de dolor crónico es el aumento de tamaño de los riñones o del hígado. El dolor crónico (4-6 semanas) afecta al $60 \%$ de pacientes con PQRAD. A menudo es un dolor severo, con un gran impacto en la actividad física y las relaciones sociales, y a menudo de difícil manejo ${ }^{4}$.

La revisión bibliográfica demuestra que la aproximación a la realidad de pacientes con esta patología desde una perspectiva enfermera es muy escasa y se fundamenta en la vivencia del dolor, las limitaciones implícitas y su manejo en la vida diaria ${ }^{5}$.

En la literatura encontrada se refiere que la situación de dolor crónico implica un impacto significativo en la calidad de vida de los pacientes, con una tendencia a presentar ansiedad y depresión, así como en sus familias, relaciones sociales y vida sexual, y en la decisión de tener descendencia ${ }^{6}$. Conceptos como la psiconefrología abarcan la repercusión psicológica de la enfermedad en este tipo de pacientes ${ }^{7}$.

Tras la realización de un primer trabajo de investigación realizado en el año 2007 por el Departamento de Enfermería de la Fundación Puigvert, que evidenció la presencia de dolor crónico, que alteraba la vida cotidiana y la dinámica familiar, se abrieron líneas de investigación cualitativa para profundizar en una mejor comprensión de la realidad subjetiva del paciente poliquístico, englobando no solo la gestión del dolor sino todo su universo emocional y de toma de decisiones ${ }^{8}$.

Con la finalidad de desarrollar intervenciones enfermeras que nos permitan mejorar la atención a estos pacientes en base a las inquietudes expresadas en primera persona por las personas afectas, nos planteamos el presente trabajo.

El objetivo de esta investigación es conocer y comprender la experiencia vital de las personas con PQRAD y las repercusiones sentidas en todos los ámbitos de su vida cotidiana, desde la perspectiva bio-psico-social y espiritual.

\section{Pacientes y Método}

El estudio se plantea bajo una óptica cualitativa según el paradigma metodológico de la Teoría Fundamentada creada en los años 60 por Glaser y Strauss y cuyo ob- 
jetivo es crear teorías que expliquen el fenómeno estudiado ${ }^{9}$. Dicha teoría va unida en este caso al paradigma constructivista que permite descubrir y explicar por el método inductivo los significados que otorgan las personas en relación a un fenómeno para comprenderlo.

La Teoría Fundamentada tiene un reconocido rigor científico que permite a los profesionales de la enfermería contextualizar el cuidado y comprender mejor la experiencia subjetiva de las personas para proporcionar una atención holística y competente ${ }^{10}$. Además es un diseño de investigación adecuado cuando existe escaso conocimiento sobre un fenómeno.

La pregunta de estudio fue: ¿Cómo afecta la PQRAD en el día a día de los pacientes atendidos en nuestro centro?

En lo referente a la muestra, los sujetos a estudio se obtuvieron del total de pacientes con PQRAD visitados en Fundación Puigvert, por el Grupo específico en la atención a los pacientes afectos de Enfermedades renales hereditarias. Disponemos de base de datos actualizada.

Se consideraron aquellos pacientes con PQRAD diagnosticada que cumplían los siguientes criterios de inclusión:

Personas con PQRAD diagnosticada; pacientes menores o igual a 45 años con ERC estadios I, II, IIIA, IIIB (de menor a mayor gravedad), que realizan controles periódicos y/o con antecedentes de crisis quística, atendidos en urgencias $y / 0$ ingreso hospitalario; pacientes de 45 a 60 años con ERC estadios II, IIIA, IIIB y IV, que realizan controles periódicos y/o antecedentes de crisis quística con atención en urgencias y/o ingreso hospitalario.

Los criterios de exclusión de la muestra fueron los pacientes con alteraciones cognitivas incapaces de comprender o participar en el estudio, los pacientes con otras enfermedades graves físicas o psíquicas, así como miembros de la misma familia en un grupo focal. Tampoco se incluyeron los pacientes que no otorgaron consentimiento informado CI.

El muestreo es intencional. El tamaño de la muestra se determinó al llegar a la saturación de los datos. Se formaron dos grupos: el primero de edades comprendidas entre los 38 y los 45 años, con ERC estadio I, II, IIIA, IIIB tratándose de cuatro varones y tres mujeres y un segundo grupo de edades comprendidas entre los 46 y los 53 años, con ERC estadio II, IIIA, IIIB siendo los componentes tres varones y dos mujeres.

El primer grupo estuvo formado por personas activas laboralmente. La mayoría tienen hijos y alguno de ellos ya ha sido diagnosticado de poliquistosis. En general tienen una vida muy activa e incluso uno de los participantes ha corrido en maratones. Solo uno de ellos tiene una actitud notoriamente distinta y en el transcurso de la reunión va reconociendo más abiertamente las limitaciones por la patología y su influencia en la vida diaria. Se trata de una persona con poliquistosis hepatorenal, siendo estos quistes hepáticos los que influyen en una peor calidad de vida con una gran afectación física y psíquica.

En el segundo grupo también se trata de personas muy dinámicas en activo profesionalmente y con trabajos en muchos casos estresantes. En general tienen familia e hijos, incluso alguno ya está diagnosticado de poliquistosis. Se repite el patrón del primer grupo en cuanto a la actitud ante la vida, siendo en su mayoría deportistas, aficionados a realizar excursiones y viajar. En este grupo dos miembros padecen poliquistosis hepatorenal bastante avanzada siendo muy limitantes para sus actividades diarias.

El instrumento para la recogida de datos utilizado fue la técnica de los grupos focales. Esta técnica es una de las herramientas básicas de la investigación cualitativa. La interacción permite producir datos que serían menos accesibles sin la interacción del grupo. Las actitudes y los puntos de vista sobre un determinado fenómeno no se desarrollan aisladamente, sino en interacción con otras personas ${ }^{11}$.

Se realizaron dos grupos focales en enero de 2017. Se llevaron a cabo en una sala tranquila del hospital, aislada para evitar interrupciones y garantizar la confidencialidad de la información. Los grupos focales fueron moderados por uno de los investigadores y con la presencia de otro de ellos para la recogida mediante el Diario de campo de la información proveniente de las actitudes y la comunicación no verbal.

Por cada grupo focal se realizó una única sesión de unas dos horas de duración. Dispusimos de un pequeño guión para que no quedara ningún tema pendiente de los que consideramos esenciales que se abordaran en el grupo. Inicialmente se abordaron cuestiones generales como temas personales y profesionales para entrar posteriormente en aspectos más específicos como el dolor, la toma de decisiones por la cronicidad de la enfermedad, 
las dinámicas familiares (por la general afectación de más de un miembro en la unidad familiar), los puntos de apoyo (familia, instituciones y profesionales a su cargo), y acabando por las expectativas de futuro.

En los últimos minutos de cada reunión se resumieron las principales ideas que habíamos tratado, exponiendo a los participantes nuestras primeras impresiones de lo percibido en el encuentro, mostrando todos ellos un gran interés por el estudio y en conocer a su término las conclusiones del mismo.

Los participantes se mostraron muy agradecidos por haber participado en el estudio y haber conocido a otras personas con su misma patología. La necesidad de compartir vivencias en el encuentro fue muy notoria a la vez de ser muy importante para todos ellos sentirse escuchados en todas aquellas inquietudes que no siempre pueden compartir sobre su enfermedad crónica.

Las conversaciones fueron grabadas y transcritas en su totalidad para el posterior análisis, lo cual fue especificado en la hoja informativa al paciente que acompañó al Consentimiento Informado que firmaron los participantes.

El análisis de los datos se ha desarrollado con la finalidad de identificar los elementos que conforman la realidad del fenómeno a estudio, describir la relación entre ellos y sintetizar el conocimiento resultante. Llevamos a cabo análisis del discurso manualmente, con una lectura exhaustiva y repetida de la transcripción de las conversaciones de los grupos focales, detectando categorías y sub-categorías de conceptos emergentes agrupándolos en temáticas y estableciendo una relación con el conocimiento obtenido en otros estudios anteriores con la idea de refutar alguna de las ideas ya extendidas o crear nuevo conocimiento sobre el tema.

En referencia a los aspectos éticos, esta investigación fue aprobada por el Comité de Ética de Investigación Clínica (CEIC) de la Institución, en fecha de 29 de noviembre de 2016. Se proporcionó a los participantes Hoja de información del estudio y Hoja de Consentimiento Informado escrito (CI).Se destacó el hecho de la voluntariedad y se garantizó la posibilidad de abandonar en cualquier momento el estudio garantizando que este hecho no debía cambiar su situación actual, ni su futura atención en el centro.

Se garantizó la confidencialidad de los datos en base a la ley 15/1999 de Protección de Datos de Carácter Personal. El acceso a los datos quedó restringido al ámbito de los investigadores. Las grabaciones fueron transcritas íntegramente, hecho explicado claramente a los participantes, asegurando la destrucción de las cintas una vez finalizado el estudio.

\section{Resultados}

El análisis del discurso de los participantes durante los grupos focales implicó la detección de cinco grandes categorías conceptuales: vivencia/mecanismos de afrontamiento de la enfermedad, repercusiones percibidas del factor hereditario, síntomas físicos y calidad de vida, perspectivas de futuro y expectativas del entorno sanitario. Dichas categorías se dividen a su vez en distintas subcategorías que abarcan un amplio espectro de ideas y de conceptos emergentes (Tabla 1).

A nivel interno, existe una percepción de enfermedad grave pero sin compromiso vital por la existencia de tratamientos sustitutivos, así como de enfermedad minoritaria con poca presencia a nivel social, en los medios de comunicación e incluso en el entorno sanitario (docencia e investigación) (Tabla 2).

La principal causa de limitación en la vida diaria es el dolor crónico, especialmente en la poliquistosis hepatorenal. Los mecanismos de afrontamiento abarcan aceptación/negación de la enfermedad mientras es asintomática. Se le otorga importancia a vivir el presente, y la búsqueda de información presenta ciclos variables de búsqueda y evitación, y una duplicidad de fuentes entre médico e Internet. La visita médica de control es vivida desde perspectivas muy diversas, que abarcan desde la tranquilidad/confianza hasta la curiosidad, inquietud 0 miedo por los resultados obtenidos (Tabla 3 ).

Los participantes hacen énfasis en la importancia de un buen autocuidado y estilo de vida, que abarca acciones como el control de la tensión arterial, adherencia terapéutica, realización de ejercicio físico moderado, reducción del estrés, seguimiento dietético y control de peso $y$, sobre todo, una adecuada ingesta hídrica como factor clave de nefroprotección.

En cuanto a la vivencia de la enfermedad a nivel externo, destacan dos posiciones contrapuestas: por un lado el silencio/ocultación de su situación a su entorno familiar, social y laboral, mientras que otras personas optan por comunicar abiertamente su situación, normalizándola. En cuanto a la primera postura, existe una voluntad de no hacer sufrir a su familia (especialmente a los hijos), no crear sentimientos de lástima en su entorno 
Tabla 1. Categorización general.

\begin{tabular}{|c|c|c|}
\hline Categorías Generales & Subcategorías (1) & Subcategorías (2) \\
\hline $\begin{array}{l}\text { 1.Vivencia y mecanismos } \\
\text { de afrontamiento. }\end{array}$ & $\begin{array}{l}\text { Nivel interno. } \\
\text { Nivel externo. }\end{array}$ & $\begin{array}{l}\text { Percepción de la enfermedad. Gravedad/ } \\
\text { cronicidad. } \\
\text { Enfermedad minoritaria. } \\
\text { Afrontamiento. Vida diaria: normalidad/ } \\
\text { limitaciones. Aceptación/negación. Vivir el } \\
\text { presente. Búsqueda de información. La visita } \\
\text { médica. Autocuidados y estilos de vida. } \\
\text { Silencio/ocultación vs comunicación/norma- } \\
\text { lización: relaciones familiares y sociales. El } \\
\text { entorno laboral. } \\
\text { Proyección de la imagen exterior. }\end{array}$ \\
\hline $\begin{array}{l}\text { 2. Repercusiones } \\
\text { del factor heredirario. }\end{array}$ & $\begin{array}{l}\text { Vivencia familiar: abuelos, padres, } \\
\text { hermanos. } \\
\text { El debut: la mutación de novo. } \\
\text { Toma de decisiones respecto a los hijos. } \\
\text { Sentimientos de transmisión de la } \\
\text { enfermedad. }\end{array}$ & $\begin{array}{l}\text { La decisión de procrear. } \\
\text { Afrontamiento de la enfermedad } \\
\text { de los hijos. }\end{array}$ \\
\hline 3. Síntomas físicos y calidad de vida. & Dolor, cansancio y otros síntomas. & Repercusión en la actividad cotidiana. \\
\hline 4. Perspectivas de futuro. & $\begin{array}{l}\text { Tratamientos sustitutivos. } \\
\text { Investigación farmacéutica: expectativas. } \\
\text { La idea de la muerte. }\end{array}$ & $\begin{array}{l}\text { Diálisis: aceptación/rechazo. } \\
\text { El trasplante de vivo como conflicto } \\
\text { personal Temor al doble trasplante hepato- } \\
\text { renal. } \\
\text { Esperanza/escepticismo. }\end{array}$ \\
\hline 5. Expectativas entorno sanitario. & Necesidad de orientación y escucha. & $\begin{array}{l}\text { Abordaje multidisciplinar. El rol de Enfer- } \\
\text { mería. }\end{array}$ \\
\hline
\end{tabular}

Tabla 2. Percepción de la enfermedad.

"La veo grave, al final si no existiera la diálisis la palmamos" (P1:1)

"No es como un cáncer, que a lo mejor lo veo mucho más grave" (P1:1)

"No nos morimos, de momento. Y luego tienes el trasplante" ( $P 1: 1)$

"Es algo que no tiene remedio...diálisis, trasplante y punto. No te mueres, no es un tumor que te puedes morir" (Pl:2)

"...no Ilamarle enfermedad cuando hablo con mi hija...tenemos unos riñones un poco deformados...el vocabulario es muy importante. No quiero hablar en términos de enfermedad y entonces, vida normal" (P5:1)
"Tú por la calle no oyes hablar de esta enfermedad (...) Ahora, un cáncer, lo que quieras, la hepatitis, lo que quieras. La nuestra es muy raro oir algo" (P7:1)

"Si fuera traumatología, eso sí que evoluciona superavanzado" (P7:1)

"Demasiado pequeña, no hace ruido nuestra enfermedad todavía" (P7:1)

"(...)Con esto que hacen el estudio genético, parece una enfermedad que no estudian mucho(...) "(P1:1) 
Tabla 3. Mecanismos de afrontamiento.

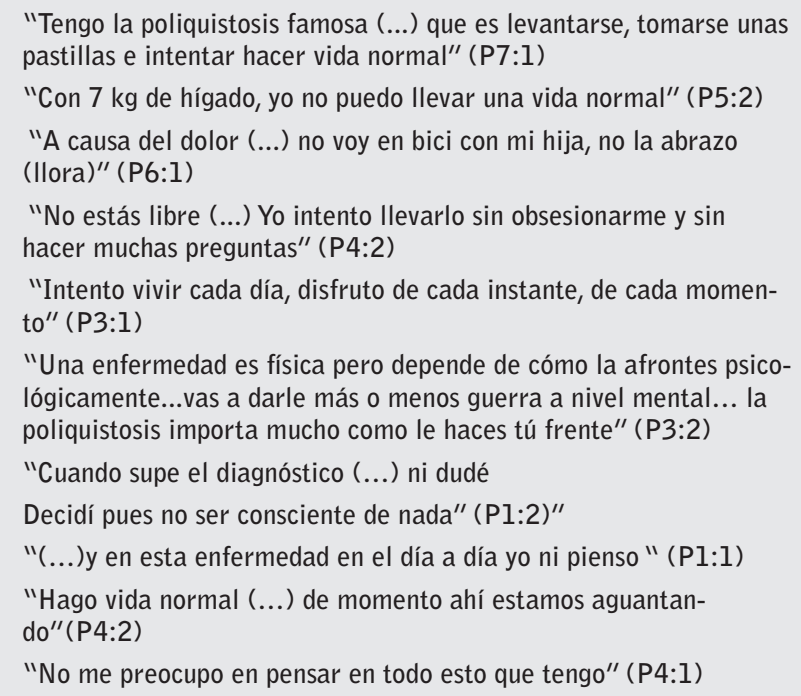

"Tengo la poliquistosis famosa (...) que es levantarse, tomarse unas pastillas e intentar hacer vida normal" (P7:1)

"Con 7 kg de hígado, yo no puedo llevar una vida normal" (P5:2)

"A causa del dolor (...) no voy en bici con mi hija, no la abrazo (Ilora)" (P6:1)

"No estás libre (...) Yo intento llevarlo sin obsesionarme y sin hacer muchas preguntas" (P4:2)

"Intento vivir cada día, disfruto de cada instante, de cada momento" (P3:1)

"Una enfermedad es física pero depende de cómo la afrontes psicológicamente...vas a darle más o menos guerra a nivel mental... la poliquistosis importa mucho como le haces tú frente" (P3:2)

"Cuando supe el diagnóstico (...) ni dudé

Decidí pues no ser consciente de nada" (P1:2)"

"(...)y en esta enfermedad en el día a día yo ni pienso " (P1:1)

"Hago vida normal (...) de momento ahí estamos aguantando"(P4:2)

"No me preocupo en pensar en todo esto que tengo" (P4:1)

"Yo le di la espalda a la enfermedad. Pero mucho, porque no me daba sintomatología" (P1:2)

"Como que no va conmigo" (P1, P3:2)

"Ha ido in crescendo el caso que le hago. 0 sea, de no hacerle ni maldito caso (...) y ahora llevo un tiempo empezando a informarme vía Internet" (P3:1)

"Lo vivo un poco como allá atrás" (P4:1)

"Cuando voy a la doctora, más que nada tengo curiosidad" (P3:2)

"Cada vez que he ido a la revisión anual, he ido en pánico"(P5:2)

"Cuando yo me enteré que tenia esta enfermedad (...) simplemente continué" (P3:2)

"Yo intento no obsesionarme" (P4:2)

"Hagamos cosas que a lo mejor mañana no puedes" (P2:2)

"Que a todo el mundo le pasan cosas (...) y a otra persona le pasa otra cosa ¿no?" (P2:1) social, y evitar consecuencias negativas como el despido o la amenaza a su carrera profesional (Tabla 4).

Por lo que se refiere a las repercusiones del factor hereditario, la mayoría de participantes relata un contexto familiar afectado por la enfermedad y la vivencia cotidiana. Ha habido dos casos de mutación de novo, en que el participante ha debutado dentro del sistema familiar, y este hecho se achaca a una cuestión de mala suerte o de azar "después de saber que nadie de la familia lo tenía (...) bueno, me ha tocado la china" (P3:1)
En cuanto a si el factor hereditario ha afectado a la decisión de procrear, ninguna familia participante lo ha considerado un obstáculo para tener hijos biológicos. Sin embargo, en un caso en que el varón era portador, la pareja decidió recurrir a una inseminación de donante para evitar la herencia genética.

El afrontamiento de la enfermedad de los hijos y qué medidas de control o seguimiento hay que tomar ha evidenciado dos actitudes: preocupación temprana versus posponer el seguimiento a la mayoría de edad de los hijos.

Tabla 4. Ocultación vs normalización.

"Yo lo tengo totalmente tapado (...) El 100\% de lo que a mí me pasa no lo sabe nadie. Nadie. No se lo digo a nadie" (P6:1)

"Lo hablo lo mínimo. Porque como estamos con la espada de Damocles, parece que lo hablas más y peor te encuentras. Cuando lo hablo me encuentro fatal" (P6:1)

"De cara a mis hijos intento minimizarlo, no quiero que vivan en un estado que tienes una madre enferma" (P2:2)

"No me veo en el día de decir: hijo, siéntate que te voy a explicar algo"(P3:2)

"En casa (...) yo no tengo nada, tengo mal en la barriga, y no abraces a la mama porque le duele y ya está, ya está (llora)" (P6:1)

"Por lástima, pobrecita (...) y que estén encima tuyo (...) A mí esto me angustia, la verdad es que no me deja dormir" (P7:1)

"Una vez lo insinué de jovencita en uno de los trabajos y cuando vi la reacción de la persona que le explicaba, pensé: estás cometiendo un error, nunca más hagas eso" (P2:2)

"Negarme un posible ascenso". "Que me echen, directamente es normal" (P6:1)
"Yo lo digo abiertamente, o sea no tengo ningún problema, lo digo y la gente lo sabe" (Pl:1)

"Yo hablándolo y explicándolo pues me desahogo, hablas con la gente, lo explicas" (P5:2)

"En casa saben el $50 \%$ de la historia (...) La historia real solo la sabe mi pareja actual. Y eso un poco...te angustia bastante" (P7:1)

"Nosotros lo tenemos integrado en la familia" (P3:1)

"Confieso que hace poco que tengo esta pareja (...) Se lo dije porque no es ético ni correcto esconder algo (...) temores a que me dijera que se buscaba a una más joven, más guapa y más sana" $(\mathrm{Pl}: 2)$

"Me levanto cada mañana pensando que somos unos pequeños héroes (....) no quiero que nadie me tenga lástima"

"No somos bichos raros (risas) ni llevamos una marca (risas)" (P3:1)

"Como ya les he dado algún susto en la oficina que han tenido que correr al servicio médico, pues ya lo he explicado" (P3:1) 
En general no existe un sentimiento de culpa por la transmisión, sino un deseo de algunos participantes de haber estado mejor informados en cuanto a métodos de reproducción que la hubieran podido evitar. A pesar de ello, el balance siempre es positivo respecto al hecho de haber tenido hijos, aunque sean portadores (Tabla 5).

El dolor es un síntoma que aparece relacionado con la calidad de vida de quien lo refiere, en los casos de intensidad moderada-alta. Los participantes relatan que "yo no puedo dormir boca abajo. No puedo soportar el dolor que me entra" (P6:1) "a mí el riñón derecho parece que lleve una pelota de rugby" (P7:1). Algunas personas sienten $\tan$ solo molestias que no son tan limitantes y explican que "más que dolor es molestia, presión" (P7:1) o que "cuando tengo dolor, estoy de baja. Es como si tuviera lumbago" (P5:2).
Otro síntoma físico ampliamente referido es el cansancio habitual y verbalizan que "el cansancio sí, creo que es la única cosa físicamente que siento" (P5:1), "hay días que te encuentras muy chafado, que no tienes ganas de nada, y aquél día han de ser comprensivos" (P4:1) o "el hígado me da muuuucho cansancio y no puedo con mi alma" (P5:2).

En cuanto a los síntomas físicos, también aparecen los cólicos, las infecciones de orina, mareo, edemas, hematuria y síntomas digestivos. En este último grupo destacan relatos como "tengo diverticulitis, he tenido varios episodios, y yo ahora mismo estoy mucho más amargada" (P2:2), "tengo una sensación de angustia siempre (...) Estoy mal, incluso sin comer" (P2:2) o "yo tengo muchos síntomas digestivos, tengo la sensación siempre de plenitud" (P2:2).

Tabla 5. Repercusiones del factor hereditario.

"Me dijeron que tenía un 25\% de posibilidades de que mi hijo tuviera la enfermedad, pero no era motivo suficiente para decidir no tener hijos" (P5:2)

"A nosotros no nos condicionó, Siempre tienes el miedo de ostras, que no le vaya a pasar pero no" (P4:1)

"Yo no sabía que se podía hacer (in vitro de donante), me enteré luego...si lo hubiéramos sabido, teníamos que haberlo preguntado" (P1:1)

"Pienso que me equivoqué (...) fueron dos hijos in vitro y no pedí (selección de embriones)"(P3:2)

"Hicimos la inseminación porque si lo hacía yo sí o sí les tocaba la china (...) al fin y al cabo padre es el que se despierta por la noche, y el que cuando está constipado está allí" (P3:1)
"Yo ahora me preocupo por mi hija de 7 años (...) le tomaron la presión y salía 11 y pico...esto sí que me sabía mal, empezar a medicarse ahora" (P1:1)

"El problema es mi hijo que está tomando pastillas para la tensión desde que era pequeño" (P4:2)

"El varón sí que lo tiene ya desde muy pequeñito, 9 años, que es lo que más me ha preocupado"(P4:2)

"A mi hija no la pienso mirar hasta que cumpla los 18 años para que no le influya como a mí me influyó en su momento" (P6:1)

"Yo no me planteo culpabilidades, ni siento. Tuve a mis hijos y punto. Ya está" (P4:1)

"Saber que le he pasado la enfermedad a mi hijo mayor. Eso me preocupa y me siento culpable. $Y$ también me hace pensar en cómo se sintió mi madre" (P3:2)

"La opción era no tenerlos, pero no los hubiera conocido"(P3:2)
La perspectiva de futuro la refieren en parte, ligada a los tratamientos sustitutivos y sus implicaciones personales. La diálisis produce reacciones de aceptación y rechazo, en función del individuo, de sus vivencias familiares y de sus miedos. Asimismo, emerge en algunos participantes la existencia de un conflicto en cuanto a la donación de vivo y un temor al doble trasplante hepatorenal, por su complejidad.

Por otra parte, la posibilidad de un fármaco que frene la progresión es percibida con esperanza y escepticismo al mismo tiempo (Tabla 6).

La idea de la muerte aparece en las conversaciones grupales de una forma muy soslayada, evitando nombrarla directamente, en frases esporádicas como "a mí lo que me enfada, no me preocupa, me enfada, es tener que dejar de ver a mis hijos o perderme una parte de su vida... esto no quiero que sea así" (P3:2) o "no quiero no conocer a mi nieto" (P3:2).

Respecto a las expectativas del entorno sanitario, emerge un sentimiento de soledad que se refleja en frases como "yo agradezco mucho esta reunión para que nos tengan un poco acogidos, porque a veces estás un poco como un zombi, deambulando, y desde visita a visita pasa a lo mejor un año...y que se nos escuche un poco más a la gente que tenemos esta enfermedad" (P7:1).

Expresan una necesidad generalizada de orientación y escucha desde una atención multidisciplinar "necesitaría algo más multidisciplinar, un tratamiento integral, 
Tabla 6. Perspectivas de futuro.

"¿Que un día tengo que hacer diálisis? Pues empezaremos a mirar si en la oficina se puede poner la máquina" (P3:1)

"Si entro en diálisis, cómo montarlo para no perjudicar al entorno porque sufren más ellos" (P1:2)

"A mí me angustia si entras en diálisis, no es agradable porque has de estar allí enchufado"( $\mathrm{Pl:1)}$

"A mí que me digas que tengo diálisis no es un consuelo, es una tortura" (P5:2)

"En mi caso que como tengo pánico a la diálisis" (P5:2)
"Mi mujer me dice 'yo no quiero que entres en diálisis, ya te lo daré yo'. Pero mira, esto sí que no me hace gracia (...) le pasa cualquier cosa..." ( $\mathrm{Pl}: 1)$

"Lo que no hemos hablado es el tema de aceptar o no un riñón de mi mujer. Es una cosa que un poco me atormenta" (Pl:1)

"Me agobia un montón, porque entonces ya en vida le debo la vida" (P7:1)
"Lo que cuesta es que un medicamento funcione o demuestre que funciona (...) Inglaterra lo paga, muchos países lo pagan y España no" (P6:1)

"Lo que me preocupa es que a mi hija le avance muy rápido y la doctora dice que para ellos seguro que habrá otras cosas" (P7:1)

"Desde que tengo más dolores, sí que realmente tengo ganas de que llegue ese medicamento" (P6:1)

"Siempre recordaré una frase de la doctora (...) para ti no hay nada. Tus hijos... (...) tengo que luchar por ellos, sí que puede haber una medicación" (P3:2) ¿no? De todo lo que tienes o puedes tener" (P2:2), "yo realmente lo necesito, este aspecto emocional y de información, en el tema de la dieta, si lo estoy haciendo bien o no. Tengo muchas dudas, muchísimas" (P1:2).

Dentro de esta perspectiva multidisciplinar, emerge la propuesta de que enfermería desempeñe una labor de acompañamiento, escucha y asesoría, como manifiesta la idea de que "además de vernos con la doctora, ¿nos podríamos ver con alguien de enfermería? Si tenemos dudas, para comentar" (P2:2).

\section{Discusión}

La experiencia vital de las personas que nacen con una PQRAD abarca una serie de vivencias y de enfoques similares que implican un cierto grado de consenso, pero también disenso en otros aspectos que hacen cada experiencia propia e individual. Destaca en este sentido una percepción de la enfermedad como relativamente grave ya que existen terapias y tratamientos sustitutivos (TRS) que permiten seguir adelante. "Yo puede ser, porque tengo tan claro que me han de cambiar la pieza (refiriéndose al riñón), que no me estoy de nada, sin pasarme ni abusar de nada" (P3:1).

En la medida en que conocen la evolución futura hacia dichos tratamientos, tratan de vivir y aprovechar al máximo el momento presente y disfrutar de la vida aquí y ahora, en general con una actitud mental positiva. "Cuando me dijeron lo que tenia, yo tuve mi momento de pensar bueno tengo una enfermedad crónica, pero bueno ya está. Luego ya se disolvió en mi mente y seguí con mi vida normal" (P3:2).
Para prolongar la etapa en que se encuentran, previa al inicio de una TRS, la mayoría hace hincapié en la importancia de un buen autocuidado y un estilo de vida saludable, no sólo por la poliquistosis sino como forma de vida, basado en un buen seguimiento dietético, control de la tensión arterial, ingesta hídrica suficiente, ejercicio físico y control de peso, la adherencia terapéutica y una reducción del estrés en su vida cotidiana. "Sí, sí, me gusta mucho nadar e ir en bicicleta, algo que me hace sentir

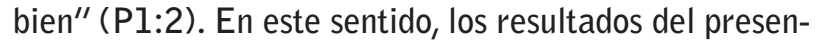
te estudio difieren en la idea de déficit de autocuidado relacionado con estados depresivos que se desprende en el artículo de Pérez Domínguez ${ }^{7}$.

En general, existe un deseo de proyectar una imagen exterior de normalidad que no produzca un sentimiento de lástima en su entorno familiar y social. "Yo también creo que es un mecanismo de defensa por mi parte, porque si yo no lo digo, la gente no me mira como enferma con lo cual yo me siento menos enferma" (P2:2). Una de las ideas que han emergido con mayor consenso entre todos los participantes es la decisión de procrear como algo indiscutible, sin que la enfermedad haya representado un obstáculo en la voluntad de tener hijos. "Igualmente yo lo he vivido como algo, que cada dos años me hacia las revisiones (...), igualmente le puede pasar cualquier otra cosa, ¿no?" (P5:1). La forma de concebirlos y la posibilidad de evitar la herencia genética a través de una fecundación de donante sí están presente, aunque de forma minoritaria. En ningún momento se cuestionan la decisión de ser padres, y el sentimiento de culpa, que tiene poca presencia, se ve compensado ampliamente. "Por eso tengo por una parte el sentido de culpa, pero por otra cuando me viene, pienso no, es una mala pasada, pero ellos van a curarse" (P3:2). Por el contrario 
otros estudios como los realizados por Heiwe y Bjuke o Phillips detectan culpabilidad por no haber realizado pruebas genéticas antes de tener hijos, y estrés emocional ante el futuro de los hijos ${ }^{5,12}$. En nuestro estudio en general, deciden vivir este tema con la normalidad que han afrontado sus propias vidas. "Quizás sigo viviendo un poco de espaldas, como yo asumí conmigo la enfermedad (...) porqué pienso que ellos van a tener más posibilida$\operatorname{des}^{\prime \prime}(\mathrm{Pl}: 2)$.

Emerge también una percepción homogénea de la vivencia familiar de la poliquistosis (abuelos, padres, hermanos, tíos) desde un recuerdo positivo, sin hacer referencia a los aspectos más negativos de la misma o de los tratamientos. La idea que subyace es de aceptación de la enfermedad dentro del sistema familiar "Mi padre se murió con 51, yo tengo 42 y él había estado en diálisis y un montón de cosas...le hicieron trasplante y tal" (P4:1).

El trasplante renal de donante vivo genera de forma unánime un cierto rechazo, y representa un conflicto personal por los riesgos que implica en el donante, e incluso temen sentir deberles la vida en vida. El doble trasplante hepatorenal suscita un cierto temor generalizado por la complejidad de la cirugía. "Y lo que sí que me preocupa mucho es el hígado, el acabar en un trasplante de hígado, más que en el riñón que lo tengo más asumido" (P2:2).

En cambio, existen algunos aspectos que son vividos de maneras muy distintas y presentan puntos de disenso importantes. Algunos participantes verbalizan una percepción de normalidad en cuanto a las actividades de la vida diaria y de cómo se sienten en relación a su enfermedad. Sin embargo, otras personas sienten que su vida está algo o muy limitada por la misma, y la causa gira en torno a la presencia de dolor físico en su día a día y las limitaciones que esto conlleva, especialmente en los casos de poliquistosis hepatorenal. "(..) me dijeron debes de tener como 7 kilos de hígado, entonces yo no puedo llevar una vida normal, lo más normal que puedo pero puedo hacer muy pocas cosas"(P5:2). Dicho aspecto corrobora la percepción descrita por Heiwe y Bjuke en su estudio, en que se describe la enfermedad como una "herencia diabólica"

Este hecho también lo observamos en un primer trabajo realizado en 2007 en Fundación Puigvert ${ }^{8}$. El dolor crónico y un aumento del volumen abdominal pueden ser muy limitantes en relación a la vida cotidiana, familiar y social, llegando a afectar anímicamente de una forma muy significativa a algunos participantes tal y como destacan Pérez Domínguez et al en su estudio ${ }^{7}$.
También aparecen diferencias importantes en cuanto a la aceptación o no de la enfermedad; algunas personas reconocen haber negado su existencia mientras se mantenían asintomáticas y otras, en cambio, refieren haberla aceptado desde el primer momento.

Destaca una divergencia muy significativa en cuanto a cómo afrontan la situación a nivel externo. En algunos casos se oculta la enfermedad de forma total o parcial al entorno familiar y social, mientras que otras personas han optado por normalizar la situación y han informado abiertamente de ella a sus allegados. Lo mismo sucede con el entorno laboral, en que el miedo a perder el puesto o a obstaculizar la carrera profesional ha llevado a algunas personas a ocultarla, mientras que en otros casos han elegido comunicarlo porque lo consideran más seguro, dada la existencia de síntomas evidentes (hipotensión, mareo, malestar).

Otra diferencia significativa radica en el afrontamiento de la enfermedad de los hijos. Mientras que en algunos casos se lleva un control médico desde la infancia, otras personas deciden postergarlo hasta su mayoría de edad, con la intención de normalizar su infancia y la vida familiar.

El sentimiento de culpa en relación a los hijos portadores aparece en algunos relatos. Sin embargo, la mayoría niega su importancia y prioriza la satisfacción de haberlos tenido. No obstante, aparece un deseo de haber recibido información sobre técnicas de reproducción para evitar la herencia genética, que hubieran valorado en su momento de haberlas conocido.

La diálisis suscita también posturas encontradas. Para algunas personas es una solución, mientras que para otras es un problema que genera ideas de rechazo por el miedo a los pinchazos o la dependencia que implica, y por el sufrimiento que pueda generar en sus familiares.

La posibilidad de que aparezca un nuevo fármaco despierta esperanza en relación al futuro de los hijos, y al mismo tiempo es vivido con cierto escepticismo porque consideran improbable que sea financiado desde el sistema público de salud debido a que, según ellos, la PQRAD se considera una enfermedad minoritaria por el conjunto de la sociedad.

Coincidimos con Pérez Domínguez et al en que faltan estudios en fases iniciales de la enfermedad, pero creemos que la necesidad de orientación y escucha dentro del sistema es una preocupación común en los participantes. Resulta crucial una atención multidisciplinar 
desde el momento del diagnóstico, en la que Enfermería apunta como uno de los pilares fundamentales en el acompañamiento, escucha y orientación de las personas con PQRAD y sus familias, desde su rol autónomo y colaborativo.

El autor declara que no hay conflicto de interés.

Recibido: 15 octubre 2017

Revisado: 28 octubre 2017

Modificado: 30 octubre 2017

Aceptado: 2 noviembre 2017

\section{Bibliografía}

1. Ars E, Bernis C, Fraga G, Martinez V, Martins $\mathrm{J}$, Ortiz $\mathrm{A}$, et al, Poliquistosis renal autosómica dominante. Guías clínicas españolas. 2015.

2. INFOGEN. México; 2013; [Consultado 10 diciembre 2015]. Disponible en http://www:// Infogen.org.mx/tag/enfermedad-renal-poliquísticaautosomica-dominante/

3. Irazábal MV, Torrs VE. Poliquistosis renal autosómica dominante. Nefrología 2001; 2: 38-51.

4. Niek F. Casteleijin, Folkert W. Visser, Joost P.H. Drenth, et al. A stepwise approach for effective management of chronic pain in autosomal-dominant polycystic kidney disease. I. Nephrology Dialisys Transplantation 2014; 29:142-153.

5. Heiwe S,RPT, Bjuke M. "An Evil Heritage":Interview Study of Pain and Autosomal Dominant Polycistic Kidney Disease"Pain Managemant Nursing.2009;10(3): 134-141.

Este artículo se distribuye bajo una Licencia Creative Commons Atribución-NoComercial 4.0 Internacional. https://creativecommons.org/licenses/by-nc/4.0/

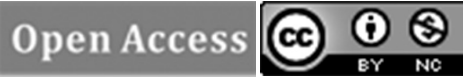

6. Expert panel recommends new approaches to management of autosomal dominant polycystic kidney disease (ADPKD). 1-4. European pharmaceutical review. [Consultado 7 diciembre 2015]. Disponible en http:// www:// europeanpharmaceuticalreview.com

7. Pérez Domínguez TS, Rodríguez Pérez $A$, Buset Rios N, Rodríguez Esparragón F, García Bello M, "et al.". Psiconefrología: aspectos psicológicos en la poliquistosis renal autosómica dominante. Revista Nefrología.2011; 31(6):716-22.

8. Calderó $M$, Font $N$, Fuenmayor $A$, Luque, $M B$, Mirada, C, Rodríguez C, et al, Atención integral al paciente con poliquistosis renal genética. Perfil clínico y experiencia vital subjetiva.Rev Soc Esp Enferm Nefrol.2007; 10(3):227-233.

9. Glaser B, Strauss A.The Discovery of Grounded Theory: Strategies for Qualitative Research. New York: Aldine; 1967.

10. Vivar G.,Arantzamendi M, López-Dicastillo 0, Gordo C, La Teoría Fundamentada como Metodología de Investigación Cualitativa en Enfermería. Index Enferm. 2010; 19(4):283-288.

11. García M M, Mateo I. El grupo focal como técnica de investigación cualitativa en salud: diseño y puesta en práctica. Aten Primaria. 2000;25:181-186.

12. Phillips A, Autosomal Dominant Polycystic Kidney Disease: A Case Study. Nephrology Nursing Journal.2009; 36(1):41-47.

13. Ávila de Tomás JF, Pajares Izquierdo, JM. Poliquistosis renal. Un caso clínico y su aproximación a través de internet.Medifam.2001; 11(9):558-561. 